\title{
SOUTH AFRICAN AIR FORCE. NOMINAL ROLL OF OFFICERS, AIR W.O.'s, AIR N.C.O.'s AND AIRMEN
}

Introduction

This contribution will take the reader back to 1922, in other words, to the time when our proud Air Force of today was still in its infancy.

It was a year in which the young South African Air Force had to struggle with a multitude of problems.

Due to the shortage of funds, it was impossible to carry out the planned work-programme in its entirety. Plans to establish a second squadron had to be abandoned. The existing organisation was reduced to two-thirds of a squadron with a corresponding reduction in the personnel at the Depot and H.Q.

The wireless section had to be dissolved and the sections for photography and armament were kept merely as skeleton sections.

The establishment allocated to the Air Force for the financial year 1922-23 consisted of 176 officers and other ranks, 54 boys and 93 Bantu labourers, while in June, 1921 the originally approved establishment necessary for the Organization Depot of the Headquarters and one squadron were respectively 270,98 and 141.

As a result of the aforementioned economy measures the approved establishment could only make available for immediate action, i.e. war service, one squadron section.

These circumstances were responsible for the fact that only one squadron could take operational action at the time of the Witwatersrand riots (10/3/1922. $15 / 3 / 1922)$ and that a member put out of action could not be replaced.

During the riots, 127 flying hours were recorded, 1 officer and 1 warrant officer were killed while 1 officer and a private were wounded.

Because of the increased demands on the reduced staff as regards organisation, construction and assembly of aeroplanes, relatively limited flight exercise was possible.

Pilots had to be employed practically full-time on administrative and technical work and during the year under review, i.e. from 1/7/1922-30/6/1923, only 701 flying hours were recorded.

A considerable section of the staff was employed in the erection of buildings such as steel hangars. Others had to strip aeroplanes kept in warehouses both in England and in the Union, they had to meticulously inspect parts and reassemble them. The average period of time necessary to make an aeroplane serviceable according to this procedure was 6 weeks, and from the time of the establishment of the South African Air Force a total of 19 aircraft had been made serviceable by the end of June, 1923, while 4 had been written off in the course of the financial year.

The names and achievements of those who devoted their initiative, ability and energy to our Air Force and who, despite adverse conditions, did their best to strengthen the existing foundations upon which we build today, must be seen in the light of those facts.

The following establishment table is therefore more than an ordinary return. Seen against the background delineated above and in the light of the 
development of our Air Force since 1922, and considering the numerous achievements after 1922 by members of the S.A.A.F., these documents will in many respects, gain a deeper significance.

\section{Inleiding}

Hierdie bydrae voer die leser in sy gedagtes terug na 1922, m.a.w. na die beginjare van die bestaan van ons trotse Suid-Afrikaanse Lugmag wat op dié tydstip nog in die spreekwoordelike kinderskoene gestaan het. worstel het.

Dit was 'n jaar waarin die jong lugmag met velerlei vraagstukke ge-

Weens geldskaarste was dit onmoontlik om die beoogde werksprogram ten volle uit te voer. Planne om ' $n$ tweede eskader op te rig, moes laat vaar word. Die bestaande organisasie is tot twee-derdes van ' $n$ eskader verminder en as ge. volg hiervan is ' $n$ ooreenstemmende vermindering in die depot- en hoofkwartierpersoneel aangebring.

Die radio-afdeling het in die slag gebly en die afdelings vir fotografie en bewapening is slegs as kerneenhede in stand gehou.

Die sterkte wat aan die lugmag toegestaan is vir die finansiële jaar 1922-1923 was 176 offisiere en minderes, 54 seuns en 93 Bantoe-arbeiders, terwyl die aanvanklik in Junie 1921 goedgekeurde sterkte wat benodig was vir die Organisasiedepot van die Hoofkwartier en een eskader onderskeidelik 270, 89 en 141 was.

As gevolg van die reeds vermelde besuinigingsmaatreëls was die goedgekeurde sterkte slegs voldoende om een eskader-afdeling vir onmiddellike optrede, d.w.s. oorlogsdiens, beskikbaar te hê.

Hierdie omstandighede het daartoe gelei dat daar ten tye van die onluste aan die Witwatersrand slegs een eskader operasioneel kon optree (10/3/192215/3/1922) en dat 'n lid wat buite geveg gestel is, nie vervang kon word nie.

Tydens die onluste-tydperk is 127 uur gevlieg, 1 offisier en 1 adj.-offisier het gesneuwel, terwyl 1 offisier en 1 manskap gewond is.

Weens die swaar eise wat aan die verminderde personeel gestel is m.b.t. organisasie, konstruksie en die montering van vliegtuie, kon daar betreklik min vliegoefeninge onderneem word. Vlieëniers moes feitlik voltyds vir administratiewe en tegniese werk aangewend word en tydens dié onderhawige verslagjaar, d.w.s. van 1/7/1922-30/6/1923, is daar slegs 701 vliegure aangeteken.

' $n$ Aansienlike deel van die personeel is vir die oprigting van geboue, soos staalvliegtuigskure, aangewend. Ander moes vliegtuie wat sowel in Engeland as in die Unie 'n geruime tyd in pakhuise gebêre was, uitmekaar haal, al die onderdele noukeurig ondersoek en inmekaar sit. Die gemiddelde tyd om een vliegtuig, ooreenkomstig hierdie prosedure, in diens te stel, was 6 weke en sedert die toestandkoming van die Suid-Afrikaanse Lugmag is daar, tot die einde van Junie 1923, altesame 19 vliegtuie in diens gestel, terwyl daar in die loop van die genoemde boekjaar 4 afgeskryf moes word.

Binne hierdie raamwerk van omstandighede moet die name en prestasies gesien word van dié wat gedurende dié tydperk hul inisiatief, vernuf, energie en daadkrag aan ons lugmag gewy en - ten spyte van stremmende omstandighede --. hul bes gedoen het om die bestaande fondamente waarop ons tot vandag toe voortbou, te verstewig.

Die sterktetabel wat hierna volg, is - gesien in bogenoemde lig - dan ook meer as 'n gewone opgaaf. Gesien teen die agtergrond wat hierbo geskets is 
en in die lig van die ontwikkeling van ons lugmag na 1922 en met die oog op die talryke prestasies wat na 1922 deur ' $n$ aantal van die toenmalige S.A.L.M.-lede behaal is, kry hierdie dokumente, in velerlei opsigte, 'n dieper betekenis.

SOUTH AFRICAN AIR FORCE, NOMINAL ROLL OF OFFICERS.

- Colonel. Sir Pierre van Ryneveld.

K.B.E., D.S.O., M.C.

Lt. Colonel. Hoare.

Lt. Colonel. Van der Spuy.

C.B.E.

F. R. G.

Major.

Welch.

] Major. Porteous.

Captain. Cloete.

Captain. Meintjes.

] Lieutenant. Holthouse.

M.C.

K. R.

Lieutenant. Wood.

[ Lieutenant. Lawson.

M.B.

I.

M.C., A.F.C.

H. B.

M.C., A.F.C.

D.

Lieutenant. Rose.

] Lieutenant. Daniel.

] Lieutenant. Daniel.

Lieutenant. Hamman. (Sick Leave).

Lieutenant. Papenfus.

D.F.C.

D.F.C.

H.

J.

S. M.

G. E. B.

C. G.

M.C., A.F.C. H. C.

Lieutenant. Venter.

D.F.C.

J. J. C.

Lieutenant. Gray.

D.F.C.

M. T. S.

Lieutenant. van der Byl.

] Lieutenant. Schoeman.

C. $\mathbf{J}$.

W. M. R.

A. L. M.

- Proceeded to Cape Town 9:5:22. Left Cape Town for South West African Protectorate 25:2:22.

] Left for South West African Protectorate 25:2:22.

[ Left for South West African Protectorate 1:6:22.

Colonel:

Director of Air Services.

Air Directorate.

6th June 1922.

SOUTH AFRICAN AIR FORCE. NOMINAL ROLL OF AIR W.O.'s, AIR N.C.O.'S AND AIRMEN.

Name

\begin{tabular}{rllll} 
No. & \multicolumn{1}{c}{ Rank } & & \\
120. & Sgt/Major & Conquer. & M.C. & E. \\
Q. & Sgt/Major & Edwards. & & E. F. \\
Q. & Sgt/Major & Robertson. & T. B. \\
$\mathbf{6 2}$. & C.A.Art. & Hellyer. & D.C.M. & F. J. \\
4. & C.A.Art. & Ottley. & & O. \\
27. & Flt./Sgt. & Blamire. & J. \\
28. & Flt./Sgt. & Browne. & H. G. P. \\
3. & Flt./Sgt. & Bunting. & C. E. H. \\
19. & Flt./Sgt. & Hutchinson. & J. \\
29. & Flt./Sgt. & Ireland-Low. & J. \\
2. & Flt./Sgt. & Parker. & & W. J. \\
39. & Flt./Sgt. & Turnbull. & D.C.M. & T. J.
\end{tabular}


No.

Rank

Name

- S/Sergt.

- S/Sergt.

- S/Sergt.

Q. Sergt.

Q. Sergt.

50. Air/Sgt.

58. Air/Sgt.

21. Air/Sgt.

1. Air/Sgt.

25. Air/Sgt.

33. Air/Sgt.

66. Air/Sgt.

93. Air/Sgt.

26. Air/Sgt.

11. Air/Sgt.

44. Air/Sgt.

22. Air/Cpl.

20. Air/Cpl.

127. Air $/ \mathrm{Cpl}$.

41. Air/Cpl.

23. Air/Cpl.

48. Air/Cpl.

42. Air $/ \mathrm{Cpl}$.

60. Air/Cpl.

98. Air/Cpl.

79. Air/Cpl.

24. Air/Cpl.

84. Air/Cpl.

34. Air/Cpl.

71. Air/Cpl.

12. Air/Cpl.

81. Air/Cpl.

96. Air/Cpl.

45. Air $/ \mathrm{Cpl}$.

63. Air/Cpl.

57. Air/Cpl.

103. Air/Mech.

Q. Private.

112. Air/Mech.

90. Air/Mech.

116. Air/Mech.

104. Air/Mech.

141. Air/App.

113. Air/App.

124. Air/Mech.

52. Air/Mech

Q. Apprentice. Buntin.

107. Air/App. Bustin.

Q. Private. Cameron.

134. Air/Mech. Carstens.

123. Air/Mech. Chambers.
F. J.

W. H.

P.

R. V.

W.

H. E. V.

S. J.

H. J.

W. G. L.

J. B.

J. B.

$\mathrm{H}$.

D.

W. H.

G. $\mathbf{M}$

P. V.

E.

C. A.

C. W.

C.

H. T.

A.

T. H.

H. P.

P. J.

R. G.

W. D.

S. D.

E. C.

F. J.

B. J.

A.

D.

A. T.

A.

M. E.

H. C.

J. H.

F. R.

P. J.

A. S.

D.

F. J.

C.

T. C.

P. J. G.

P. A.

G.

F.

J. C.

P. J. 
No. Rank Name

37. Air/Mech. Coetzee.

118. Air/Mech. Collins.

111. Air/Mech. Coode.

106. Air/Mech. Cooper.

40. Air/Mech. Cowley.

61. Air/Mech. Crofton.

131. Air/Mech. Cross.

67. Air/Mech. Crossley.

47. Air/Mech. de Beer.

145. Air/Mech. de Bruyn.

J. H.

H. E.

R. L.

H. G.

S. I.

G. P.

A. $\mathbf{R}$.

R. C.

O. S.

117. Air/App. Drury.

114. Air/App. du Plessis.

$\mathrm{T}$.

80. Air/Mech. du Plessis.

G. A. B.

C. J.

J. P. S.

142. Air/Mech. Dyason.

C. B.

Q. Apprentice. Ensor.

G. E.

102. Air/Mech. Flowers.

H. F.

85. Air/Mech. Forbes.

91. Air/App. Ford.

139. Air/Mech. Frost.

N.

P. A.

C. N.

55. Air/Mech. Gardner.

E. $\mathrm{H}$.

78. Air/App. Grace.

95. Air/Mech. Green.

J. D'A.

L.

130. Air/Mech. Green.

L. B.

138. Air/Mech. Grundlingh.

J. C.

77. Air/Mech. Hadley.

G.

140. Air/Mech. Harvey.

J. M.

H. J.

128. Air/Mech. Heddle.

C.

133. Air/Mech. Hollings.

R. J.

115. Air/Mech. Innes.

M.M.

A. $S$.

119. Air/App. Jackson.

P. J.

30. Air/App. Lawrenson.

C. R.

38. Air/Mech. Lawson.

C.

99. Air/Mech. Lawson.

L. S.

51. Air/Mech. Liebenberg.

C. J.

110. Air/Mech. Liebenberg.

J. J. S.

100. Air/App. Mayhew.

143. Air/Mech. Mare.

121. Air/Mech. McKenzie.

J. A. C.

A.

A.

16. Air/Mech. Meyer.

46. Air/Mech. Meyer.

J. G.

8. Air/Mech. Moore.

J. G.

144. Air/Mech. Negus.

J.

97. Air/Mech. Nel.

D.

101. Air/Mech. Padbury.

P. J.

85. Air/Mech. Parker.

G. S.

147. Air/Mech. Pea.

H. J.

132. Air/Mech. Pollock.

A.

P. G.

65. Air/Mech. Porter.

R. J.

35. Air/App. Preller.

125. Air/Mech. Rickards.

V. W.

E. $\mathbf{R}$. 
68. Air/Mech. Ridgers. W. R

138. Air/App. Slater. R. H.

82. Air/Mech. Steiger. W. R.

36. Air/Mech. Sterley. A. E.

109. Air/Mech. Stone.

94. Air/Mech. Strode.

74. Air/Mech. te Brugge.

135. Air/Mech. Terblanche. P. J.

10. Air/Mech. Thompson. A. G.

129. Air/Mech. Trapler. D.

59. Air/Mech. Upton-Smith. F. E.

49. Air/Mech. van der Linde. H. J.

17. Air/App. van Noort. F. C. H.

15. Air/Mech. van Straaten. C. A.

6. Air/App. Vermaak. M. T. S.

18. Air/Mech. Vermaak. J. M.

92. Air/Mech. Verney. W. C.

137. Air/Mech. van der Westhuizen. H. N.

146. Air/Mech. van Schalkwyk. G. P. K.

54. Air/Mech. Ware.

7. Air/App. Wilson. A.

108. Air/App. Wimble. N. C.

89. Air/Mech. Woodward. H.

31. Air/App. Zeederberg. W. H. 


\section{OPSOMMING}

In die inleiding is die toestand, waarin die jong Suid-Afrikaanse Lugmag in 1922 verkeer het, kortliks geskets. As gevolg van besuinigingsmaatrë̈ls is die uitbreiding van die S.A.L.M. vertraag.

Die vernaamste gedeelte van die bydrae is die sterktetabel van S.A.L.M. personeel op 6 Junie 1922. Onder die name kom 'n aantal voor as staatmakers wat, in die daaropvolgende jare, letterlik en figurlik, tot groot hobgtes uitgestyg het.

\section{SUMMARY}

In the introduction, the condition in which the young South African Air Force found itself in 1922, is briefly sketched. As a result of economy measures the development of the South African Air Force was retarded.

The major portion of the contribution is the establishment table of the S.A.A.F. on 6 June, 1922. Amongst the names given one finds several of the pioneers who were to rise to great heights, literally and figuratively, in the years to come. 